\title{
Fabricación de electrodos para control de transporte y alineamiento a micro y nanoescalas usando técnicas bottom-up y top-down
}

\author{
Darwin Rodríguez ${ }^{1}$, Alba Ávila²
}

'Servicio Nacional de Aprendizaje SENA

22 Departamento de Ingeniería Eléctrica y Electrónica, Universidad de los Andes darwindubay@misena.edu.co, a-avila@uniandes.edu.co

\begin{abstract}
Resumen
El continuo avance de aplicaciones en dispositivos de autoensamble, posicionamiento, sensores, actuadores, y que permitan controladamente la manipulación de micro y nanoestructuras, han generado amplio interés en el desarrollo de metodologías que permitan optimizar la fabricación de dispositivos para el control y manipulación a micro y nanoescalas. Este proyecto explora técnicas de fabricación de electrodos con el fin de encontrar una técnica óptima y reproducible. Se compara el rendimiento de cada técnica y se describen protocolos de limpieza y seguridad. Se diseñan e implementan tres geometrías para movilizar y posicionar micro y nanopartículas de hierro en una solución de aceite natural. Finalmente se generan campos eléctricos a partir de electroforesis, con el fin de encontrar la curva que describe el desplazamiento de las partículas con respecto al potencial aplicado. Estos resultados generan gran impacto en los actuales esfuerzos de fabricación bottom-up (controlando con campos la ubicación) y la movilidad en dispositivos electrónicos. El hecho de fabricar geometría planar con electrodos genera la posibilidad de que se pueda integrar movimiento de partículas a los circuitos integrados que se fabrican en la actualidad.
\end{abstract}

Palabras clave: micropartículas, nanotecnología, microelectrodos, electroforesis, hidrofobicidad.

Editora: Pataquiva-Mateus, A. Y.

Citation: Rodríguez, D. y Ávila, A. (2014). Fabricación de electrodos para control de transporte y alineamiento a micro y nano escalas usando técnicas bottom-up y top-Down. Revista Mutis 4(2).

Received: September 15, 2014; accepted: December 6, 2014; Published on line: December 31, 2014

Copyright: $\odot 2014$ Rodríguez, D. y Ávila, A. This is an open-access article, which permits unrestricted use, distributions and reproduction in any medium, provided the original author and source are credited

Competing Interests: The authors have no conflict of interest.

\section{Manufacture of electrodes for transport con- trol and alignment micro and nano scales using techniques bottom-up and top-down}

\begin{abstract}
The continued application progress in auto-assembly devices, positioning, sensors, actuators, and controllably allow manipulation of micro and nanostructures. Those have generated wide interest in the development of methodologies to optimize the control manufacturing devices and handling micro and nanoscales. This project explores electrode-manufacturing techniques in order to find an optimal and reproducible technique. Performances of each technique are compared and cleaning and security protocols are described. Three geometries to mobilize and position micro and nano
\end{abstract}


iron particles in a solution of natural oil were designed and implemented. Finally, electric fields are generated from electrophoresis to find the curve that describes the particles movement relative to applied potential. These results generate great impact on current manufacturing efforts bottom-up (controlling with golf location) and mobility in electronic devices. Finally, they were made that planar geometry electrodes, it generates the possibility of particles movement are included to integrated circuits manufacturing today.

Keywords: microparticles, nanotechnology, microelectrodes, electrophoresis, hydrophobicity.

\section{Introducción}

En la actualidad existen avances en aplicaciones de micro y nanomanipulación y abundante literatura que aborda temas de movimiento y control de posición de micro y nanopartículas, allí se estudian fenómenos físicos que generan movimiento y control de posición de células biológicas, virus y proteínas (Burke, 2003), existen también metodologías de manipulación que se basan en la generación de campo magnético para generar actuación en dispositivos a microescalas aplicándolo a microrrobótica (Croquette, Charlie \& Vincent, 2002). En el caso de manipulación de micropartículas usando luz láser con longitud de onda de $\lambda=532 \mathrm{~nm}$ es posible la construcción de micromanipuladores que aprovechen este fenómeno físico (Dholakia, Reecea \& Gub, 2007). Recientemente se han utilizado las ondas de ultrasonido para generar control de partículas en medios líquidos viscosos (Zurich, 2009), métodos basados en campos magnéticos y métodos ópticos, que requieren de instrumentación compleja, mientras que metodologías basadas en el uso de campo eléctrico permiten resaltar la integración del estímulo para mover el elemento con el propio elemento que convierten el uso de campos eléctricos en una forma sencilla de manipular micro y nanopartículas. Algunos autores usan la fuerza eléctrica con un fenómeno conocido como dielectroforesis (DEP) aplicadas en electrodos donde las fuerzas debidas al campo eléctrico son relevantes para el estudio del desplazamiento y control de posición (Burke, 2003). Adicionalmente las aplicaciones en el transporte de biomateriales (Huang, et al., 2001), alineamiento de nanotubos de carbono (Cicoria \& Sun, 2008), y ordenamiento de nanocables (Hesketh, Gallivan, Kumar, Erdy \& Wang, 2005), genera metodologías de clasificación y alineación que son útiles para estudiar la utilidad de este fe- nómeno. La generación de diferentes matrices de electrodos a diferentes espacios y escalas permite analizar el comportamiento de velocidad y fuerza eléctrica concluyendo que a mayor escala de la geometría menor es la velocidad a la que se desplaza la partícula (Bligh, Stanley, Hubbard, T \& Kujath, 2008).

En este documento se exploran técnicas de fabricación de electrodos, se generan protocolos, que tiene en cuenta la seguridad (Hampshire, 2009), manejo de espacios de laboratorio como sala limpia y laboratorio de microscopía, con el fin de obtener un método óptimo para fabricar patrones y geometrías; adicionalmente se probaron técnicas de fabricación como: rasgado de material que aprovecha la fuerza mecánica para romper la capa conductora generando espacios aislantes, Electron Beam que usa el haz de electrones para fabricación, litografía suave que aprovecha las propiedades poliméricas del polydimethylsiloxane (PDMS) para generar moldes de geometrías, fotolitografía con Aligner que usa máscaras y SF100 que no usa máscaras, exponiendo a luz ultravioleta $(\lambda=400 \mathrm{~nm})$ para imprimir patrones prediseñados. Al ensayar los métodos mencionados se encontró que la técnica de fotolitografía sin máscaras usando SF-100 resulta pertinente para la fabricación de prototipos. Haciendo uso de esta, se fabricaron tres geometrías basadas en patrones lineales y uniformes, que buscan la concentración de campo eléctrico manteniéndolo constante durante el recorrido de la partícula, estas geometrías fueron probadas para mover a microescalas. A continuación se describen las condiciones óptimas de caracterización de electrodos aprovechando el fenómeno de electroforesis que genera el movimiento de partículas de hierro previamente caracterizadas en forma, tamaño y distribución (Nurmi, et al., 2004). Estas partículas son sumergidas en aceite natural que luego de aplicar diferentes potenciales en los electrodos durante un intervalo de tiempo se determina el desplazamiento de las partículas usando microscopía óptica como instrumento de observación y medición.

Finalmente se obtienen resultados como curvas de voltaje vs. desplazamiento, voltajes de ruptura, voltajes de iniciación, sensibilidad y criterios de durabilidad de los electrodos fabricados.

\section{Materiales y Métodos}

A continuación se describe la metodología y técnicas de fabricación exploradas, y detalles de los procedimientos, donde en la primera etapa se exploran 5 
técnicas de fabricación y en la segunda se analizan el desplazamiento de micro y nanopartículas de hierro depositadas sobre tres geometrías fabricadas con el método optimo hallado en la primera etapa.

\section{Técnicas de fabricación exploradas y me- todología}

En la figura 1, se muestra el desarrollo del proyecto que involucra fases y secuencias, allí se denota una etapa inicial correspondiente a pruebas de técnicas de fabricación y otra etapa que involucra el movimiento debido a la electroforesis sobre tres geometrías de micropartículas de hierro suspendidas en aceite natural.

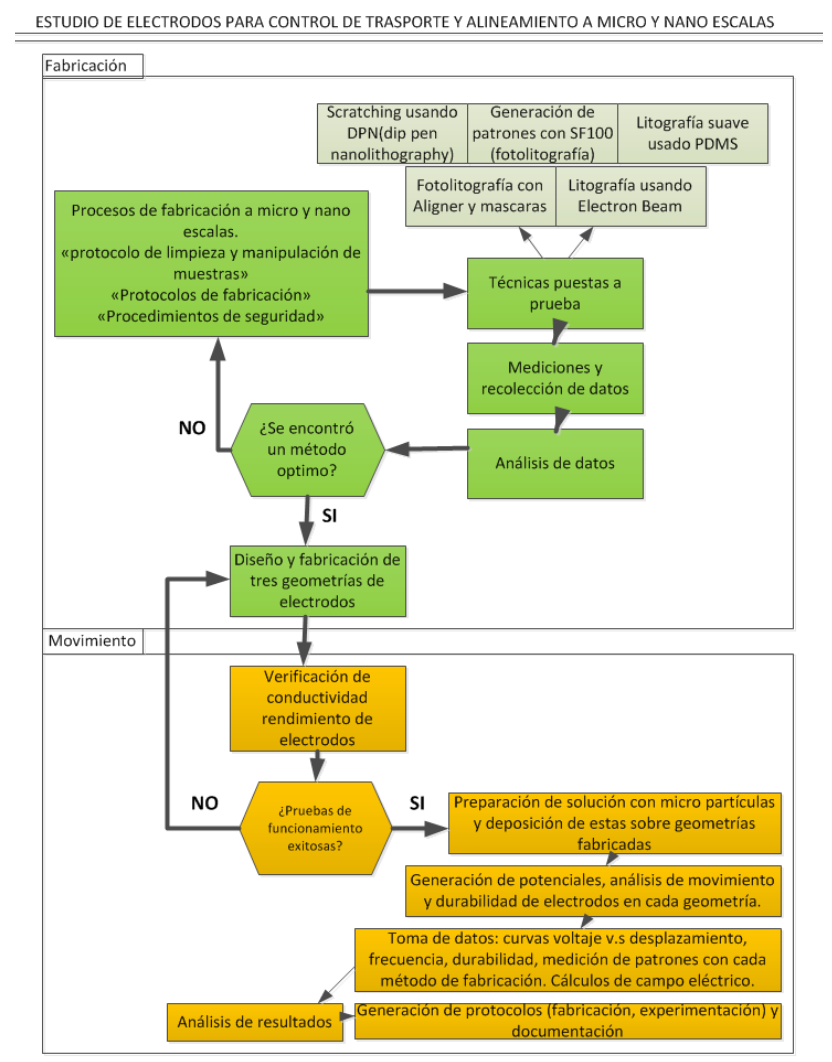

Figura 1. Procedimientos, secuencias y fases del proyecto

Para la selección de las técnicas de fabricación que se sometieron a prueba, se tuvo en cuenta la disponibilidad de técnicas, equipos y laboratorios tanto en la Universidad de los Andes como en el Sena (TecnoParque y TecnoAcademia Nodo Cazucá), a continuación se enuncian las técnicas de fabricación a micro y nanoescalas estudiadas (Nanolnk, 2010). Estas se comparan según la dirección de escala de trabajo (top-down, bottom-up, paralelo) y se listan los equipos utilizados, esto es resumido en la tabla 1.

Tabla 1. Dirección de escala de trabajo para cada técnica de fabricación

\begin{tabular}{|c|c|c|c|c|}
\hline Técnica & $\begin{array}{l}\text { Top- } \\
\text { down }\end{array}$ & $\begin{array}{c}\text { Bot- } \\
\text { tom-up }\end{array}$ & Paralelo & Equipos \\
\hline $\begin{array}{l}\text { Fotolitografía con } \\
\text { Aligner (Godoy, } \\
\text { Ruy, Ávila \& } \\
\text { Rodríguez, 2012) }\end{array}$ & $x$ & & $x$ & Aligner \\
\hline $\begin{array}{l}\text { Litografía con } \\
\text { Electron Beam } \\
\text { (MicroChem, } \\
\text { 2009) }\end{array}$ & $x$ & & & $\begin{array}{c}\text { SEM } \\
\text { (Scanning } \\
\text { Electron } \\
\text { Microscope) }\end{array}$ \\
\hline $\begin{array}{l}\text { Fotolitografía } \\
\text { con SF100 } \\
\text { (Schiltz, 2011) }\end{array}$ & $x$ & & & SF-100 \\
\hline $\begin{array}{l}\text { Litografía Suave } \\
\text { (Duffy, et al., } \\
\text { 1998) }\end{array}$ & $x$ & $x$ & $x$ & PDMS \\
\hline $\begin{array}{l}\text { Rasgado } \\
\text { con Dip Pen } \\
\text { Nanolithography, } \\
\text { (Piner, 1999) }\end{array}$ & $x$ & $x$ & $x$ & $\begin{array}{l}\text { NLP2000/ } \\
\text { DPN5000 }\end{array}$ \\
\hline
\end{tabular}

\section{Técnicas de fabricación}

Se exploraron 5 técnicas de fabricación que son descritas a continuación, para cada una se tomaron mediciones de patrones con el fin de encontrar información cuantitativa que permita compararlas.

Evaluar reproducibilidad y repetibilidad de patrones que tienen pistas conductoras requieren la deposición de películas delgadas metálicas, estas películas delgadas se fabricaron usando deposición física con el equipo PVD (Physical Vapor Deposition) Edward 301 disponible en sala limpia de la Universidad de los Andes. A continuación se describe cada técnica iniciando con los protocolos de limpieza y seguridad.

\section{Protocolos de limpieza y seguridad}

Los procesos de fabricación requieren de procedimientos minuciosos en cuanto a seguridad, limpieza de muestras y sustratos, en esta sección se describen los procedimientos de limpieza, que incluyen materiales y métodos, adicionalmente de las precauciones de 
seguridad y equipos de protección usados en el momento de manipular sustancias y equipos involucrados en las técnicas de fabricación.

En la figura 2 se muestra el proceso de limpieza usado en los procesos de fabricación, adicionalmente se tienen en cuenta utensilios de protección personal como guantes, tapabocas, gafas de protección UV (ultravioleta), delantal industrial y hojas de seguridad MSDS (Material Safety Data Sheet) de las sustancias y reactivos involucrados en los procesos, se siguieron las recomendaciones de seguridad descritas en las fichas de seguridad MSDS.

\section{Rasgado usando el NLP2000}

Usando la plataforma de nanolitografía NLP2000 (Dip pen nanolithography system from nanoink) disponible en el Sena, en TecnoParque y TecnoAcademia Nodo Cazucá, se procede a rasgar o rayar sobre una película delgada metálica desbastando las áreas donde no se requiere capa conductiva, esta técnica es distinta a las demás ya que remueve el material de forma mecánica y no de forma química, se utilizaron películas delgadas de oro $(100 \mathrm{~nm})$ sobre un trozo de $1 \times 1 \mathrm{~cm}$ de silicio (Intrínseco, <100>). Adicionalmente se probaron líneas sobre películas delgadas de aluminio (100 nm) sobre un trozo de vidrio de $1 \mathrm{~cm} \times 1 \mathrm{~cm}$ de vidrio. Los resultados de ancho de la perforación se obtienen de medidas tomadas en varias líneas, los datos y estadísticas son resumidos en la tabla 2 donde se aprecia la media, varianza y error relativo conforme al valor esperado de $1 \mu \mathrm{m}$ en el grosor de línea. Esta técnica demuestra poca homogeneidad y no garantiza la repetibilidad de líneas. Durante las pruebas se programó la plataforma para recorrer 10 trazos con espacios de $20 \mu \mathrm{m}$ a una velocidad de $1 \mu \mathrm{m} / \mathrm{s}$ (ver figura 3 ), se tomaron 15 mediciones lo largo de cada trazo. Estas mediciones fueron realizadas con microscopio óptico y de fluorescencia (Carl Zeiss A1) debidamente calibrado usando micrómetro ocular.

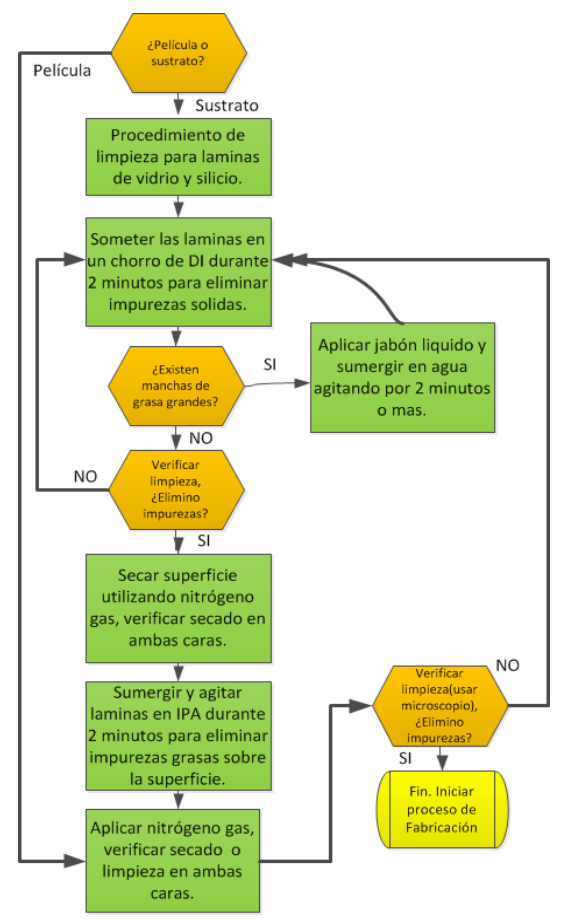

Figura 2: Protocolo de limpieza para películas y sustratos

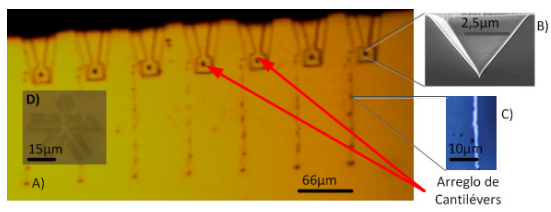

Figura 3. Imágenes de procesos con NLP2000 A)Scratching sobre película de oro (100 nm) sobre silicio intrínseco(100), con pasos de $10 \mu \mathrm{m}$ a una velocidad de piezo motor de $1 \mu \mathrm{m} / \mathrm{s}$ en Y. B)Punta del cantiléver tomada con SEM (Nanolnk, 2010) . C) fotografía usando microscopio óptico de trazo realizado. D) escudo Sena impreso con técnica DPN.

Tabla 2. Datos estadísticos obtenidos usando técnica de rasgado

\begin{tabular}{|c|c|c|c|c|}
\hline Trazo & Media $(\mu \mathrm{m})$ & Desviación estándar ( $\mu \mathrm{m})$ & Varianza $(\mu \mathrm{m})^{2}$ & Error relativo \\
\hline 1 (oro) & 1,73 & 0,83 & 0,65 & $73 \%$ \\
\hline 2 (oro) & 1,18 & 0,86 & 0,70 & $18 \%$ \\
\hline 3 (oro) & 1,87 & 1,37 & 1,76 & $87 \%$ \\
\hline 4 (oro) & 2,4 & 0,69 & 0,45 & $140 \%$ \\
\hline 5 (oro) & 2,67 & 0,97 & 0,87 & $167 \%$ \\
\hline 6 (aluminio) & 1,86 & 0,78 & 0,57 & $86 \%$ \\
\hline 7 (aluminio) & 2,5 & 0,71 & 0,46 & $150 \%$ \\
\hline 8 (aluminio) & 2,45 & 0,66 & 0,41 & $145 \%$ \\
\hline 9 (aluminio) & 2,76 & 0,73 & 0,50 & $176 \%$ \\
\hline 10 (aluminio) & 2,56 & 0,63 & 0,37 & $156 \%$ \\
\hline
\end{tabular}




\section{Fotolitografía con Máscaras-Aligner}

En este proceso se diseñaron y fabricaron máscaras por dos métodos, el primero usando una mesa reductora DeVere 480 para reducir patrones hasta los $20 \mu \mathrm{m}$ de resolución usando la materiales y metodologías similares a las usadas en fotografía convencional (Godoy et al., 2012). El segundo método consiste en imprimir máscaras usando Photoploter, equipo que imprime sobre acetato fotosensible a 8000 dpi (equivale a una distancia entre punto de $3.17 \mu \mathrm{m}$ ). Las máscaras fueron puestas a prueba estampando patrones sobre películas de aluminio (100 nm) sustrato vidrio de $2 \times 2 \mathrm{~cm}$ con óxido de indio (ITO) depositado sobre un sustrato flexible de $2 \times 2$ $\mathrm{cm}$ respectivamente. Las películas fueron cubiertas por una capa de fotoresist SC1827 por Spin coating.

A)

B)

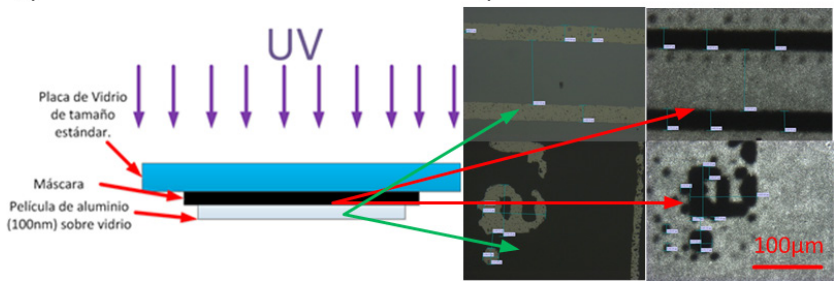

Figura 4. Montaje para fotolitografía A) Montaje de máscaras y sustrato en Aligner Karl Suss MJB. B) Patrones sobre aluminio (100 nm) vidrio y máscaras método fotográfico en acetato

Estas fueron expuestas a luz UV ( $\lambda=400 \mathrm{~nm})$ a $14,9 \mathrm{~W} /$ $\mathrm{cm}^{2}$ de potencia, el ataque de aluminio se realizó con hidróxido de tetrametilamonio (3,5\%, agua desionizada (DI)). La figura 4 ilustra el proceso de exposición y estampado de patrones usando equipo de fotolitografía (Aligner Karl Suss MJB), allí se muestra la ubicación de sustrato y máscara. La tabla 3 muestra resultados estadísticos de 27 mediciones tomadas de máscara y patrón de aluminio en tres máscaras distintas. Adicionalmente se realizaron mediciones sobre películas de óxido de indio (ITO) generando un error relativo medio de $26,8 \%$.

Tabla 3. Resultados estadísticos de comparación máscara-patrón impreso usando fotolitografía

\begin{tabular}{lcccc}
\hline Película/máscara & $\begin{array}{c}\text { Media } \\
(\mu \mathrm{m})\end{array}$ & $\begin{array}{c}\text { Desviación } \\
\text { estándar } \\
(\mu \mathrm{m})\end{array}$ & $\begin{array}{c}\text { Varianza } \\
(\mu \mathrm{m})^{2}\end{array}$ & $\begin{array}{c}\text { Error } \\
\text { relativo } \\
\text { máximo }\end{array}$ \\
\hline Aluminio 1 & 122,77 & 40,38 & 1449,24 & \multirow{2}{*}{$18,1 \%$} \\
Máscara 1 & 122,48 & 42,49 & 1605,06 & \\
\hline Aluminio 2 & 53,62 & 71,85 & 4588,70 & $27,40 \%$ \\
Máscara 2 & 68,56 & 70,64 & 4435,56 & \\
\hline Aluminio 3 & 41,79 & 13,13 & 153,35 & $21,10 \%$ \\
Máscara 3 & 38,46 & 13,87 & 170,95 & \\
\hline
\end{tabular}

\section{Litografía con Electron Beam}

Usado PMMA (polimetilmetacrilato) como resist sensible al haz de electrones que emite el Electron Beam, se usó un SEM que al controlar el encendido y apagado del filamento y enfocando en diversas áreas se formaron líneas, para este caso se usaron películas delgadas de cobre $(100 \mathrm{~nm})$ sobre vidrio cubiertas con PMMA 950A11 y 950C4 generando una película delgada de polímero de $2 \mu \mathrm{m}$ (A11) y $485 \mathrm{~nm}$ (C4) (MicroChem, 2009).

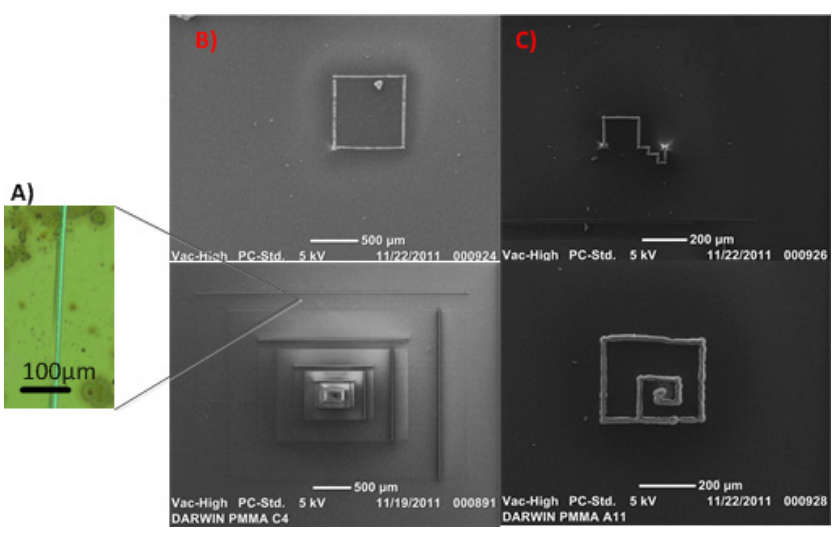

Figura 5. Imágenes de 4 geometrías con SEM A) Litografía sobre cobre (100 $\mathrm{nm}$ )-vidrio tomada con microscopio óptico. B) Geometrías de líneas usando SEM Jeol5000 con enfoque de 20.000 X a 10 kV, 15 kV sobre película cubierta con PMMA C4. C) Geometrías de líneas usando SEM sobre película cubierta con PMMA A11.

Las películas poliméricas se depositaron por spin coating, el ataque de cobre se realizó con cloruro férrico (34\%, DI). En la figura 5 se muestra la litografía realizada usando el SEM Jeol5000 disponible en el Sena que tiene una magnificación máxima de 20.000 X, lo cual limita al haz de electrones a líneas mayores de $1 \mu \mathrm{m}$, se probó con dos potenciales de filamento $10 \mathrm{kV}$ y 15 kV generando así una mayor afectación del polímero. La visualización se realiza a $5 \mathrm{kV}$ ya que un potencial elevado afectaría el polímero y deformaría la geometría hecha de forma acelerada. Cada ensayo se realizó en alto vacío (0.1 $\mathrm{mPa})$, con una configuración estándar de foco, contraste y astigmatismo. En la tabla $4 \mathrm{Se}$ tomaron 10 mediciones por cada línea impresa sobre el polímero, mediciones tomadas con microscopio óptico y valor de referencia $50 \mu \mathrm{m}$ y $20 \mu \mathrm{m}$ de grosor que son valores que se esperarían idealmente. Aunque esta técnica es usada en litografías de alta resolución $(10 \mathrm{~nm})$ no es posible hacer esto usando SEM dadas las limitaciones que posee el equipo, lo ideal para lograr litografías de mejor resolución y geometrías más complejas es usar Focuced Ion Beam (FIB). 


\begin{tabular}{ccccc}
\hline $\begin{array}{c}\text { Película } \\
\text { de PMMA }\end{array}$ & $\begin{array}{c}\text { Media } \\
(\mu \mathrm{m})\end{array}$ & $\begin{array}{c}\text { Desviación } \\
\text { estándar }(\mu \mathrm{m})\end{array}$ & $\begin{array}{c}\text { Varianza } \\
(\mu \mathrm{m})^{2}\end{array}$ & $\begin{array}{c}\text { Error } \\
\text { relativo } \\
\text { medio }\end{array}$ \\
\hline C4 & 56,67 & 0,69 & 0,43 & $13,82 \%$ \\
A11 & 22,68 & 0,64 & 0,38 & $5,74 \%$ \\
\hline
\end{tabular}

Tabla 4. Resultados estadísticos entre patrones en PMMA A11 y C4

\section{e. Generación de patrones con SF100}

La técnica usada en litografía con Aligner es similar a la trabajada en esta sección, la única diferencia es que se trabaja con el SF100 (Intelligent Micro Patterning), equipo disponible en sala limpia de la Universidad y que estampa geometrías prediseñadas en mapas de bits ( $5 \mu \mathrm{m}$ y $15 \mu \mathrm{m} \mathrm{X} \mathrm{pixel),} \mathrm{radiando} \mathrm{luz} \mathrm{UV} \mathrm{(400} \mathrm{nm)} \mathrm{sobre} \mathrm{las} \mathrm{pe-}$ lículas grabando el patrón deseado. Una de las limitaciones que tiene este equipo es que únicamente se puede imprimir un patrón al tiempo, sin embargo, aunque resulta ventajoso a la hora de fabricar prototipos no es óptimo para trabajar impresiones masivas (Schiltz, 2011).
La película de fotoresist positivo depositado por spin coating, expuesto a luz UV $(\lambda=400 \mathrm{~nm})$ a $1,7 \mathrm{~W} / \mathrm{cm}^{2}$ de potencia, y los resultados de las deposiciones sobre aluminio (100 nm) - vidrio, cobre $(100 \mathrm{~nm})$ - vidrio, aluminio $(100 \mathrm{~nm}$ ) - silicio (intrínseco <100>) y cobre $(100 \mathrm{~nm})$ - silicio (intrínseco $<100>$ ), son presentados en la figura 6 . La tabla 5 resume los resultados obtenidos luego de tomar 10 datos por cada línea.

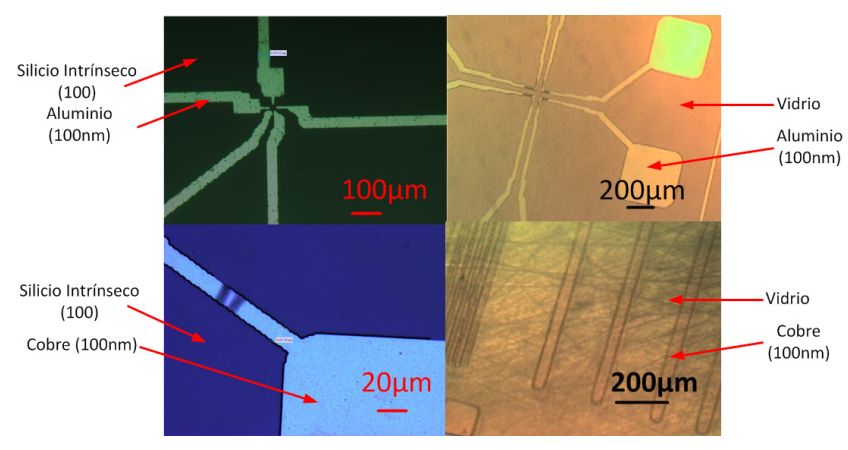

Figura 6. Geometría y patrones estampados en aluminio y cobre sobre sustrato de silicio intrínseco $<100>$ y vidrio

Tabla 5. Resultados estadísticos usando técnica de fotolitografía con SF100

\begin{tabular}{|c|c|c|c|c|}
\hline Líneas impresas sobre muestras & Media $(\mu \mathrm{m})$ & Desviación Estándar ( $\mu \mathrm{m})$ & Varianza $(\mu \mathrm{m})^{2}$ & Error relativo medio \\
\hline Línea 1- 10 datos sobre aluminio (65 $\mu \mathrm{m})$ & 65,05 & 0,35 & 0,11 & $0,1 \%$ \\
\hline Línea 2- 10 datos sobre cobre (100 $\mu \mathrm{m})$ & 100 & 0,12 & 0,01 & $0,0 \%$ \\
\hline
\end{tabular}

\section{Litografía Suave (Soft Lithography)}

Para fabricar patrones con Soft Lithography se usa PDMS, que genera un molde flexible, que luego de su curado, el patrón donde se había depositado queda estampado sobre la superficie de contacto.

Con el fin de comprobar este método, se estampa una geometría con hexágonos que son medidos tanto en el PDMS como en la geometría original con el fin de encontrar la presión y resolución.

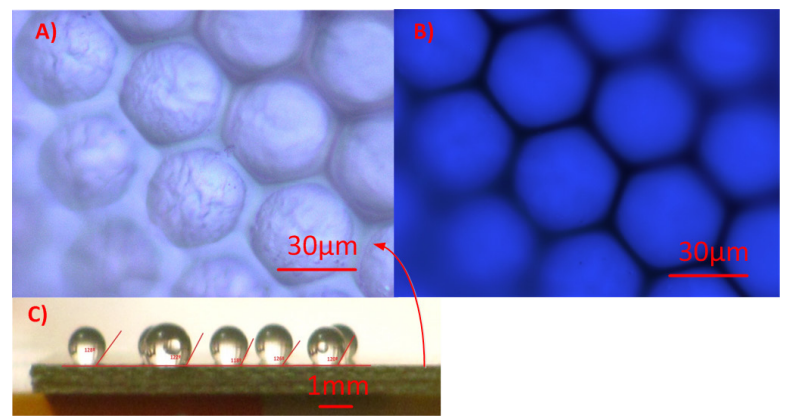

Figura 7. Imágenes de estructuras y efecto. A) Geometría de hexágonos con relieve. B) Geometría de hexágonos con huecos. C) Ángulos de contacto con gotas de DI sobre superficie hidrofóbicas
Se preparó una solución de PDMS con una relación 10:1 de prepolímero. La figura 7 muestra la geometría original y su copia (Duffy, et al., 1998). Finalmente se muestran los resultados estadísticos de 30 mediciones realizadas Tabla 6.

Tabla 6. Datos estadísticos para litografía suave con PDMS con geometrías hexagonales

\begin{tabular}{lcccc}
\hline Geometría & $\begin{array}{c}\text { Media } \\
(\mu \mathrm{m})\end{array}$ & $\begin{array}{c}\text { Desviación } \\
\text { estándar }\end{array}$ & Varianza & $\begin{array}{c}\text { Error } \\
\text { relativo } \\
\text { medio }\end{array}$ \\
\hline $\begin{array}{l}\text { Hexágonos } \\
\text { de } 20 \mu \mathrm{m}\end{array}$ & 20,02 & 0,11 & 0,01 & $0,1 \%$ \\
\hline
\end{tabular}

\section{Movimiento y alineación de partículas}

En esta sección se describe el desplazamiento de partículas de hierro sobre tres geometrías fabricadas con fotolitografía usando el SF100, técnica que demostró ser la adecuada para la fabricación de prototipos de electrodos. Inicialmente se caracterizan tamaños de partículas de hierro, seguido de la preparación de solución con aceite natural, montaje de equipos, me- 
dición de desplazamiento a diferentes potenciales y pruebas de durabilidad de geometría.

\section{Caracterización de partículas}

Se depositó polvo de hierro comercial sobre una superficie de vidrio de $2 \times 2 \mathrm{~cm}$ aplicando una gota de IPA (isopropanol) y luego de secar a la intemperie para garantizar separación se observaron en SEM, allí se realizaron mediciones de diámetros y se hallaron datos estadísticos con el fin de determinar homogeneidad de las partículas.

En la figura 8 se observan imágenes de SEM donde se ven partículas de hierro aglomeradas a diferentes escalas de observación. La tabla 7 muestra los resultados estadísticos de las partículas encontradas en diversas regiones, allí se observa que existe mayor cantidad de partículas de tamaños $<1 \mu \mathrm{m}$.

Tabla 7. Numero de partículas encontradas en 4 tomas con SEM en zonas distintas

\begin{tabular}{lc}
\hline Tamaño de partícula & Número total encontrado \\
\hline$<1 \mu \mathrm{m}$ & 77 \\
Entre $1 \mu \mathrm{m}$ y $2 \mu \mathrm{m}$ & 52 \\
Entre $2 \mu \mathrm{m}$ y $4 \mu \mathrm{m}$ & 22 \\
Entre $4 \mu \mathrm{m}$ y $8 \mu \mathrm{m}$ & 13 \\
Entre $8 \mu \mathrm{m}$ y $10 \mu \mathrm{m}$ & 3 \\
\hline \\
\hline
\end{tabular}

Figura 8. Imágenes de SEM. A) Micro y nanopartículas de hierro observadas con SEM a escala de $20 \mu \mathrm{m}$. B) Nanopartículas adheridas a micropartículas escala de $1 \mu \mathrm{m}$

\section{b. Preparación de la solución y montaje}

Tomando aceite natural a base de girasol, se mezcla $1 \mathrm{ml}$ de aceite con $10 \mathrm{mg}$ de polvo de hierro que contiene micro y nanopartículas, esto permite el desplazamiento de las partículas de hierro fácilmente y mejora la durabilidad de la solución ya que el aceite no se evapora con facilidad.
El montaje eléctrico, acople de equipo generador de potencial y microscopio óptico requirió el diseño de un sistema de dos puntas controlado por PC con posicionado automático de microcontactos. La figura 9 muestra el montaje donde se realizaron mediciones de desplazamiento de partículas.

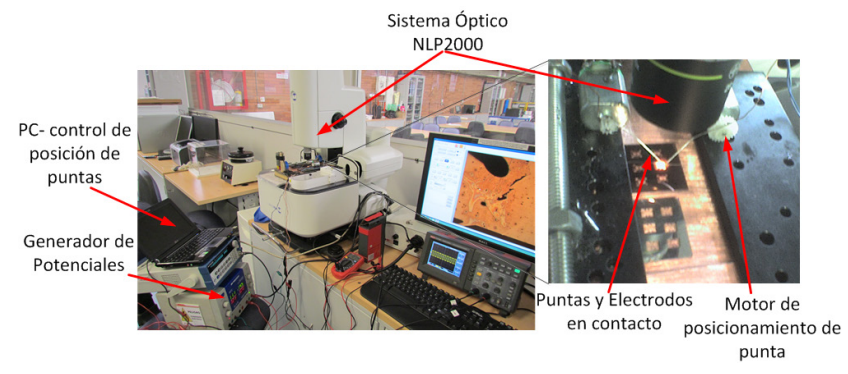

Figura 9. Montaje experimental usando NLP2000 y sistema de dos puntas. Se muestra fuente de poder y generador de señal como generador de potenciales

Adicionalmente se realizó un montaje usando BWmicroscope USB con el cual se tomaron algunas mediciones finales en conjunto con el sistema de dos puntas.

\section{Procedimiento experimental y curvas de potencial}

En la figura 10 se muestran las tres geometrías, las cuales se caracterizan por mantener formas lineales y no curvas, las formas lineales permiten mantener un campo eléctrico constante durante todo el recorrido de la partícula. Los puntos de conexión se seleccionaron por presentar proximidad, facilidad de ubicación de puntas y no obstrucción de campo visual del microscopio óptico.

Las mediciones de desplazamiento de partículas de hierro de tamaños entre $1 \mu \mathrm{m}$ y $5 \mu \mathrm{m}$ fueron tomadas por medio de microscopía óptica en cada geometría, se conectó cada electrodo a una fuente de voltaje variable, generando potenciales con intervalos de $5 \mathrm{~V}$ durante $1 \mathrm{seg}$, seguidamente el potencial es desconectado y se espera 1 minuto a que las partículas estabilicen su movimiento, finalmente se invierte la polaridad del voltaje y se aplica la misma cantidad de potencial por 1 seg y así sucesivamente para cada nivel de potencial hasta alcanzar un voltaje donde la geometría presente ruptura o llegue al valor máximo de $100 \mathrm{~V}_{D C}$. 


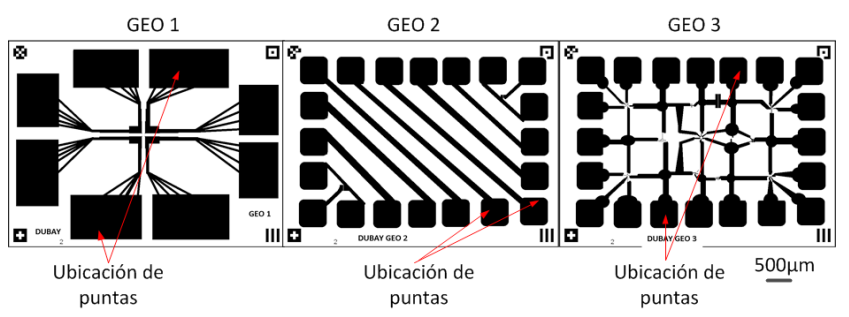

Figura 10. Esquema de distribución de los electrodos y contactos. A) Geometría 1. B) Geometría 2. C) Geometría 3

La ecuación 1 permite calcular el campo eléctrico a partir del potencial aplicado en cada geometría teniendo en cuenta el espacio que existe entre cada línea conductora, allí $V$ es el voltaje aplicado sobre el electrodo y $d$ la distancia de separación de la pista (Bligh et al., 2008).

En la tabla 8 se muestra el cálculo de campo eléctrico para potenciales de ruptura de cada geometría usando la ecuación 1 . De allí se concluye que a mayor distancia entre electrodos, mayor es el voltaje de ruptura, para que esto suceda es necesario que se genere un campo eléctrico cercano a los $900 \mathrm{kV} / \mathrm{m}$.

$$
E_{0}=\frac{V}{d} \text { Ecuación (1) }
$$

\begin{tabular}{lc}
\hline \multicolumn{1}{c}{ Geometría } & Ecuación \\
\hline Electrodos simples geometría 1. & 908,31 kV/m \\
Electrodos líneas paralelas geometría 2. (no & $386,07 \mathrm{kV} / \mathrm{m}$ \\
ruptura) & $900,34 \mathrm{kV} / \mathrm{m}$ \\
\hline Electrodos líneas concéntricas geometría 3. & 9
\end{tabular}

Tabla 8. Campo eléctrico calculado a partir del potencial de ruptura y dimensiones de cada geometría

La figura 11 muestra la respuesta de desplazamiento vs. potencial aplicado en cada geometría, donde el $(+)$ indica que se aplica un voltaje positivo, (-) indica que se aplica voltaje negativo generando un desplazamiento en la dirección contraria y cada punto de lectura tuvo un error de $10 \%$. Los datos de voltaje para la geometría 1 y 3 de la figura 10, se tomaron hasta un máximo antes de que se presentara ruptura de la pista conductora tal como se muestra en la figura 12B.

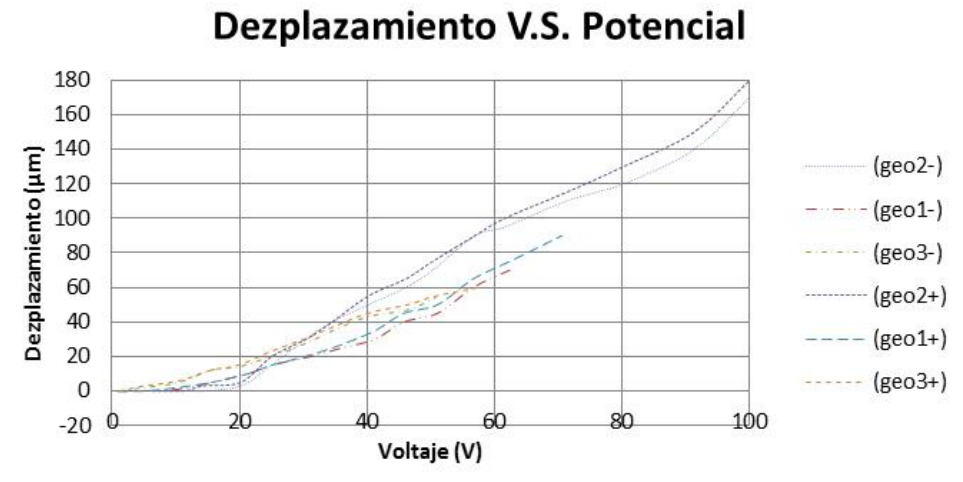

Figura 11. Curva desplazamiento vs. potencial en cada geometría para polarización positiva y negativa

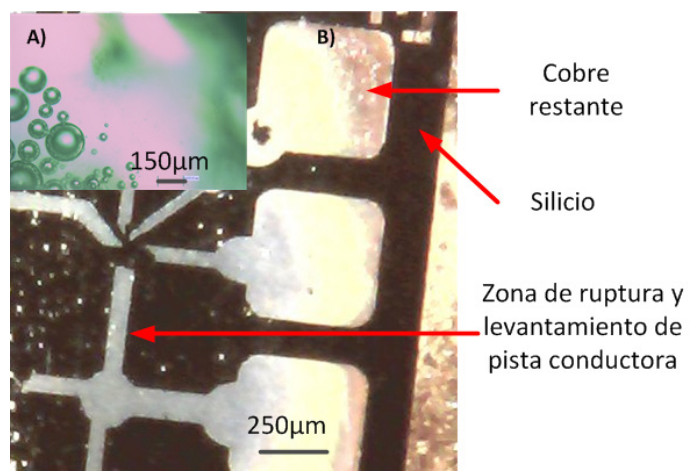

Figura 12. Imágenes de microscopía óptica. A) Burbujas generadas por calentamiento de aceite. B) Geometría 3 después de someterla a un voltaje de ruptura de $57,65 \mathrm{~V}_{\mathrm{DC}}$
En los resultados se describen los análisis relevantes.

Reusabilidad y tiempo de vida del dispositivo

Al generar un campo eléctrico demasiado grande, se genera arco eléctrico calentando la solución de aceite produciendo burbujas que destruyen los electrodos y levantan las pistas conductoras (figura 12a). En la geometría 1 se presentó un voltaje de ruptura de 70,65 $\mathrm{V}_{D C^{\prime}}$ en la geometría 3 un voltaje de $57,65 \mathrm{~V}_{D C}$ y en la geometría 2 no se presentó ruptura.

Al pasar una corriente de $40 \mathrm{~mA}$ por una pista de 50 $\mu \mathrm{m}$ de la geometría 1 se produce ruptura de pista y 
calentamiento del aceite, en lo posible y para evitar un desgaste de los electrodos, se debe evitar elevar al voltaje o corriente a niveles cercanos a condiciones de ruptura, esto alarga la vida útil del dispositivo.
En general luego de usar una geometría no es posible limpiarla o retirar el aceite, esto resulta complejo dada la adherencia a la superficie y de limpiarlo causaría deterioros en la película delgada.

\section{Resultados}

Los resultados encontrados durante la exploración de las técnicas se resumen en la tabla 9, organizadas de acuerdo a su rendimiento (repetibilidad, reproducibilidad y pertinencia para fabricación de prototipos).

Tabla 9. Resultados de técnicas de fabricación exploradas

\begin{tabular}{|c|c|c|c|}
\hline Técnica & $\begin{array}{l}\text { Error relativo } \\
\text { máximo }\end{array}$ & $\begin{array}{l}\text { Error relativo } \\
\text { medio }\end{array}$ & Características principales \\
\hline & & & Útil para realizar prototipos, medidas estándar de \\
\hline Fotolitografía sin máscaras usando SF-100 & $0,1 \%$ & $0,05 \%$ & $\begin{array}{l}5 \mu \mathrm{m} \text { x pixel, acepta diseños sin necesidad de más- } \\
\text { cara. }\end{array}$ \\
\hline Litografía suave con PDMS & $0,1 \%$ & $0,1 \%$ & $\begin{array}{l}\text { Genera molde de estructuras nanométricas. Permi- } \\
\text { te imprimir varias geometrías al tiempo. Cubre am- } \\
\text { plias áreas y dimisiones. }\end{array}$ \\
\hline Elelctron Beam usando SEM & $13,82 \%$ & $9,78 \%$ & $\begin{array}{l}\text { Limitaciones en la ubicación del rayo, permite rea- } \\
\text { lizar una geometría. Logra alta resolución con focu- } \\
\text { sed ion beam. }\end{array}$ \\
\hline Fotolitografía con máscaras usando Aligner. & $27,40 \%$ & $22,20 \%$ & $\begin{array}{l}\text { Ideal para imprimir múltiples patrones al tiempo. } \\
\text { Requiere máscaras. }\end{array}$ \\
\hline Rasgado de material con NLP2000 & $176 \%$ & $120 \%$ & $\begin{array}{l}\text { Utiliza múltiples puntas y arreglos para rasgar que } \\
\text { deben ser cambiados continuamente. Presenta } \\
\text { desgaste en las puntas, no genera trazos uniformes } \\
\text { y homogéneos, útil para deposiciones de material } \\
\text { localizado. }\end{array}$ \\
\hline
\end{tabular}

De la figura 11 se puede concluir que el cambio en la polaridad del potencial aplicado sobre las 3 geometrías incrementa el desplazamiento de partículas para potenciales positivos frente a potenciales negativos. También se evidencian los voltajes de ruptura y voltajes de iniciación para cada geometría, la geometría 2 no presentó ruptura al llegar al voltaje máximo de la fuente de $100 \mathrm{~V}_{D C}$

Se probó que el desplazamiento de las partículas es directamente proporcional al voltaje, este fenómeno se presentó en las tres geometrías diseñadas, cuando las partículas de hierro están sumergidas en aceite, esto se evidencia al analizar la curva de la figura 11 , se nota una modificación de la pendiente evidenciando mayor desplazamiento conforme aumenta el voltaje. Esto muestra que cada geometría muestra un cambio de sensibilidad diferente.

El efecto del campo eléctrico genera desplazamiento de las partículas con diámetros de entre $1 \mu \mathrm{m}$ y $5 \mu \mathrm{m}$, pero si el tamaño de la partícula aumenta, la distancia recorrida es menor estando sometida a una misma magnitud de campo.

\section{Conclusiones}

En este proyecto se exploraron técnicas de fabricación que permiten definir patrones directos sobre la superficie (rasgado) y patrones que son estampados por capas sobre la superficie (litografía).

Se encontró que la técnica de fotolitografía sin máscaras usando SF100 es más óptima en cuanto a calidad y facilidad en la fabricación. A pesar de que algunas de las geometrías fabricadas con el método de litografía suave con PDMS no resultaron ser eficientes para el movimiento de partículas, sí mostraron ser útiles en propiedades especiales como hidrofobicidad, este el caso de la geometría de la figura 7A, donde se aplican gotas de agua desionizada y se procede a medir ángu- 
los de contacto entre gota y muestra evidenciando valores mayores a 90 (figura 7C) típicos de superficies hidrofóbicas.

Durante la exploración de técnicas de fabricación, se trabajó con sustratos rígidos y flexibles y películas delgadas metálicas y de óxido de indio, que resulta útil en procesos de fabricación de dispositivos electrónicos en aplicaciones como pantallas de visualización y táctiles.

En la experiencia de fabricación se integraron protocolos de seguridad para el manejo de materiales, procesos y espacios para las geometrías diseñadas y fabricadas.

Se demostró que fabricando electrodos con tres geometrías, se logra movilizar partículas metálicas a diversos potenciales por medio de electroforesis generando resultados en cuando a durabilidad, voltajes de ruptura, voltajes de iniciación, desplazamiento de partícula y campo eléctrico.

Estos resultados pueden ser aplicados a la movilización, control y alineamiento de partículas sensibles al campo eléctrico, generando aplicación en microposicionadores, microactuadores, micro y nanomanipuladores.

Como trabajo futuro se espera evaluar estas geometrías movilizando partículas de otras materiales, microorganismos, células, estructuras vivas y gotas que ayuden al posicionamiento en biosensores, nanoporos, células o nanoalambres, así como también aplicar potenciales con frecuencia variable con el fin de encontrar modelos de posicionamiento más precisos y óptimos. Se espera explorar técnicas de fabricación como Focused lon Beam (FIB) para lograr gomerías de mayor resolución y escala nanométrica.

\section{Agradecimientos}

Se agradece al Sena (TecnoParque y TecnoAcademia) por el apoyo en el uso de infraestructura, al grupo de investigación CMUA y a la Facultad de Ingeniería de la Universidad de los Andes.

\section{Referencias}

Godoy, A., Ruy, S., Ávila, A., Rodríguez, D. (2012). Fabricación de máscaras fotolitográficas para micro- electrónica usando una cámara fotorreductora. Bogotá: Universidad de Los Andes.

Bligh, M., Stanley, K. G., Hubbard, T., Kujath, M. M. S. (2008). Two-phase interdigitated microelectrode arrays for electrokinetic transport of microparticles. Journal of Micromechanics and Microengineering, 18(5), 055007.

Burke, P. (2003). Nanodielectrophoresis: Electronic Nanotweezers. Encyclopedia of nanoscience and nanotechnology, 6, 623-641.

Croquette, Charlie, Gosse, Vincent. (2002). Magnetic Tweezers: Micromanipulation and Force Measurement at the Molecular Level. Biophysical Journal, 2(6), 3314-3329.

Duffy, D., Cooper, Mc., Olivier, J., Schueller., George, M,. Whitesides, J. C. (1998). Polydimethylsiloxane (PDMS) on SU-8 Mold. Emory University: Doctoral dissertation.

Dholakia, K., Reecea, P., Gub, M. (2007). Opical Micromanipulation. Chemical Society Reviews, 37(1), 42-55.

New Hampshire (2009). Nanomaterials safety program. University of New Hampshire. Particle and fibre Toxic, 1-8.

Hesketh, P.J., Gallivan, M. A., Kumar, S., Erdy, C. J., Wang, Z. L. (2005). The Application Of Dielectrophoresis To Nanowire Sorting And Assembly For Sensors. IEEE Intelligent Control, Proceedings of the 2005 IEEE International Symposium on, Mediterrean Conference on Control and Automation.

Huang, Y., Ewalt, K. L., Tirado, M., Haigis, R., Forster, A., Ackley, D., Heller, M. J., O'Connell, J. P., Krihak, M. (2001). Electric manipulation of Bioparticles and macromolecules on microfabricated electrodes. American Chemical Society, 73(7), 1549-1559.

MicroChem. (2009). Nano PMMA Copolymer Developer. Manual of Copolymers, 1-8.

Nanolnk. (2010). Introduction to Nanoscale Science and Technology Version 1. Skokie, Illinois USA: Nanolnk.

Nurmi, J. T., Tratnyek, P. G., Sarathy, V., Baer, D. R., Amon- 
ette, J. E., Pecher, K., Wang, Ch., Linehan, J. C., Matson, D. W., Penn, R. L., Driessen, M. D. (2004). Characterization and Properties of Metallic Iron Nanoparticles: Spectroscopy, Electrochemistry, and Kinetics. Environmental Science \& Technology, 39(5), 1221-1230.

Piner, R. D. (1999). “Dip-Pen” Nanolithography. Nanolithography science, 283(5402), 661-663.

Schiltz, D. D. (2011). Analog Lithography of complex
Phase Plates for Sub-diffraction Lithography. Le of contents, (1).

Cicoria, R., Sun, Yu. (2008). Dielectrophoretically trapping semiconductive carbon nanotube networks. Nanotechnology, 19(48), 485303.

Zurich, E. (2009). Micromanipulation of Small Particles within Micromachined Fluidic Systems Using Ultrasound. Onsernone: Diss. ETH No. 18452. 